\title{
Über das Prothallium und die Sporenpflanze von Botrychium Lunaria Sw.
}

\author{
Von H. Bruchmann.
}

Hierzu Tafel I und II.

Während von den einheimischen Ophioglossazeen Ophioglossum vulgatum eine reiche ungeschlechtliche Vermehrung durch Adventivsprosse an den Wurzeln besitzt und der Hauptsache nach sich durch diese fortpflanzt, fehlt solche Botrychium Lunaria ganz. Für die Entstehung jedes Sporophyten dieser Art ist somit stets ein Gamophyt vorauszusetzen. An dem Standort jeder sporentragenden Pflanze dieser Art und zwar in der Bodentiefe ihres Rhizoms keimte einmal eine Botrychiumspore und entwickelte das Prothallium, welches dann die uns entgegentretende Pflanze hervorbrachte. Jede Sporenpflanze lehrt somit, daß an ihrem Standorte ihre Sporen im Boden, in welchen sie durch den Regen geführt werden können, auch keimen, und man wird daher an den Standorten der Botrychiumpflanze auch deren Prothallien $\mathrm{zu}$ erwarten haben. Leider sind diese Gebilde sehr klein und nur mit der Lupe deutlich erkennbar. Auch sind ihre Keimpflänzchen kein Wegweiser zu ihrem Fundort, denn diese wachsen zuerst mehrere Jahre nur unterirdisch, und wenn sie dann ihr erstes grünes Blättchen an die Erdoberfläche führen, werden sie nicht mëhr im Zusammenhange mit dem Prothallium apgetroffen.

Irmisch entdeckte 1854 bei Sondershausen in Thüringen zuerst die eigenartigen Keimpflanzen von Botrychium Lunaria und machte Hofmeister auf dieselben aufmerksam, worauf dann beide im Oktober desselben Jahres auch die Prothallien fanden. Doch dürfte, wie aus der sehr unvollkommenen und zum Teil unrichtigen Darstellung dieser Gebilde durch Hofmeister ${ }^{1}$ ) hervorgeht, das Material wohl nur mangelhaft und nicht ausreichend gewesen sein, und von einer Wiederholung solcher Funde ist bis dahin nichts bekannt geworden.

Im Juli 1893 war Campbel1) „so glücklich“, in Grosse Ile Michigan von B. virginianum eine Anzahl alter Prothallien zu finden,

1) Ho fmeister, Abh. d. k. sächs. Ges. d. Wiss. 1857, Bd. V, pag. 657-662.

2) Campbell, Moses and Ferns, London 1895, pag. 224-228. 
die sämtlich in Verbindung mit der jungen Sporenpflanze angetroffen wurden. Sie waren aber für das Studium dieses Gamophyten ungeeignet. Jeffrey ${ }^{1)}$ dagegen fand in den Jahren 1895-97 an verschiedenen Orten Canadas (in Nordamerika) denselben Gamophyten in großer Anzahl und in allen Stufen der Entwicklung. Die Anwesenheit dieser Gebilde verrieten die grünlichgelben Keimblätter der Sporenpflanze dieser Art, und da diese großen Prothallien auch in reicher Menge bei einander zu finden waren, so konnten durch bloßes Durchsieben des Bodens reiche Ernten erzielt werden. Neuerdings macht noch Lyon ${ }^{2}$ ) bekannt, daß er im Frühjahr 1903 Prothallien von B. obliquum Muhl.; Willd. in beträchtlicher Zahl in der Nähe d. Echo in Minnesota gesammelt habe. Auch die von B. virginianum sind von ihm aufs neue an zwei Stellen gefunden worden. Die ausführliche Publikation hierüber steht bevor.

Meine Prothallienfunde von B. Lunaria machte ich an verschiedenen Stellen Deutschlands und der Schweiz. Von Sondershausen, wo Hofmeister im Verein mit Irmisch diese Körper zuerst fand, erhielt ich auf meine Anfrage nach dem Vorkommen dieser Sporenpflanze die Nachricht, daß sie dort in den letzten zehn Jahren nicht mehr gefunden sei. In der Schweiz auf den Wiesen von St. Moritz und Arosa ermittelte ich trotz fleißigen Suchens in den Sommern 1903-05 nur einzelne Prothallien und Keimpflanzen und zwar auffallend tief, 8-10 cm unter der Erdoberfläche. Die beste Ausbeute brachte mir eine Waldwiese bei Ohrdruf in Thüringen und ein gleicher Ort am Bocksberg bei Gotha, wo ich eine neue, sehr reiche Ansiedlung dieser Sporenpflanze fand.

Die Prothallien von B. Lunaria fanden sich in dem sandigen Boden der Thüringer Waldwiesen in nur geringer Tiefe, etwa 1 bis $3 \mathrm{~cm}$ unter der Erdoberfläche, in dem Wurzelgeflecht der Wiesenpflanzen vor. Sie traten mir nicht selten zu mehreren, einmal zu zehn bei einander entgegen und können in diesem im Sommer meist trockenen Erdreich einer anhaltenden Dürre, wie sie z. B. die Sommer 1903 und 04 uns brachten, erfolgreich widerstehen, denn nach den ersten Regentagen solcher Perioden fand ich sie unbeschädigt vor. In dem nassen, durch die Feuchtigkeit dunkel erscheinenden Erdreich treten die Prothallien wie auch die kleinen Keimpflanzen deutlicher hervor und können weniger leicht übersehen werden.

1) Jeffrey, The Gametophyte of Botrychium virginianum 1898.

2) Lyon, The Pteridophytes of Minnesota XX. 1903, p. 249. 


\section{Der Gamophyt.}

Hofmejster bringt nur wenig Angaben über das Prothallium. Er stellt es als eine eiförmige Masse festen Zellgewebes von etwa 1/2 Linie größtem Durchmesser dar. Außen sei es lichtbraun, innen gelblichweiß von Farbe und mit spärlichen, mäßig langen Wurzelhaaren allseitig besetzt. Seine Zellen, deren Größe vom Mittelpunkte nach der Peripherie hin abnehme, seien mit größeren und kleineren Klumpen eines halbdurchsichtigen Stoffes vollgestopft. Das Prothallium trage auf der nach oben gekehrten Seite Antheridien, die Höhlungen in seiner Masse darstellen, und auf der entgegengesetzten Seite Archegonien, welche Organe dem Prothallium vollständig eingesenkt seien. Künstlich ausgesäte Sporen keimten nicht.

Nach meinen Prothalliumfunden erscheinen mir unsere Gamophyten auch als kleine eiförmige, aber auch als herzförmige, meist etwas zusammengedrückte (also im Querschnitt elliptische) Zellkörper, weißlich oder schwach hellbraun von Farbe, von etwa 1 bis $2 \mathrm{~mm}$ Länge und $1 / 2$ bis $1 \mathrm{~mm}$ Breite, die herzförmigen mit noch größeren Ausdehnungen, alle mit langen Rhizoïden allseitig mäßig besetzt.

Ich habe versucht, auch diese sechste Art meiner Prothallienfunde, wie die fünf vorher bekanntgegebenen, zuerst in natürlicher Größe erkennbar darzustellen, dieses gelang hier nur, indem ich diese kleinen Gebilde mit ihrer Fundstelle zugleich zeichnete (Fig. 1). Auch ihre Darstellungen in dreifacher Vergrößerung (Fig. 2-6) lassen ihre äußere Gestaltung nicht erkennen, jedoch für die kleinen braunen Keimpflanzen (Fig. 7-16) dürfte sie schon genügen. Während das Prothallium von B. virginianum $\mathrm{zu}$ den größten aller bis jetzt bekannten Gamophyten gehört und bis $20 \mathrm{~mm}$ Länge und $15 \mathrm{~mm}$ Breite erreicht, ist es bei B. Lunaria auffallend klein, es gleicht jenem aber in der Form, und wahrscheinlich werden alle Arten dieser Gattung die gleiche Gestaltung besitzen, die $\mathrm{zu}$ erkennen uns für unsere Art erst durch eine 16 fache Vergrößerung möglich wird (Fig. 17-25). Die kleineren Gamophyten sind meist kugelig oder eiförmig (Fig. 17, 19 u. 20), die größeren herzförmigen (Fig. 18, 24 u. 25) erreichen in solcher Form die doppelte Ausdehnung der einfachen. Ihre langen, oft den Durchmesser übertreffenden Wurzelhaare sind am Grunde stets braun gefärbt und geben dem Gamophyten seinen lichtbraunen Anflug, der im Herbste mehr als im Frühjahre dem Beschauer entgegentritt. Außer den langen, an älteren Körperteilen meist abgestorbenen Rhizoiden finden sich auf dem Rücken auch einzelne kleine einzellige oder mehrzellige derartige GeBilde, die Paraphysen, vor. Die Prothallien sind stets monözisch. Auf 
ihrer der Erdoberfläche zugekehrten Seite tragen sie sowohl Antheridien als auch Archegonien, entgegen den Angaben Hofmeisters ${ }^{1}$ ), der letztere Gebilde auf der Unterseite gefunden haben will. Auf dem hinteren Körperteile fehlen die Geschlechtsorgane ganz. Die Antheridien treten auf der mittleren Partie des Rückens in einer unregelmäßig angeordneten Reihe meist zu mehreren bei einander auf und führen mit ihren jüngeren Formen auf die vordere noch wachsende Prothalliumregion (Fig. 27s). Ihre ältesten Formen am hinteren Teil des Gamophyten sind entleert und flach; sie können bei größeren Formen $\mathrm{zu}$ einem Kamme hervortreiben, in welchem sie $\mathrm{zu}$ zweien und auch $\mathrm{zu}$ dreien nebeneinander ausgebildet werden.

$\mathrm{Zu}$ beiden Seiten des antheridialen Rückens treten die Archegonien meist im lockeren unregelmäßig gestellten Zweizuge auf (Fig. 27ar). Ihre Achsen sind nach den Seiten schief aufwärts gerichtet, und schon nahe am fortwachsenden Prothalliumteile blühen sie und finden Befruchtung, während die Antheridien erst weiter zurück reifen und entleerte Höhlungen aufweisen.

Fast jeder Gamophyt unserer Art bringt einen, zuweilen auch zwei Sporophyten zur Entwicklung, die anfangs als geringe Hervorwölbungen nahe dem fortwachsenden Prothalliumteile erkennbar werden (Fig. 19, 20 u. $27 \mathrm{em}$ ), darauf aber mit der ersten Wurzel aus dem Inneren hervorbrechen (Fig. 21-25) und seitwärts fortwachsen, ohne daß die Ausbildung eines Stammscheitels nebst Kotyledon bemerkbar wird. Auch Keimpflanzen mit zwei Wurzeln traf ich seltener noch mit dem Mutterprothallium im Zusammenhange an. Vielfach findet man schon einwurzelige, dann aber mehrwurzelige Keimpflanzen frei im Boden vor (Fig. 6-16), und wenn deren erstes grünes Blatt über die Erdoberfläche tritt, besitzen auch sie, wie die Keimpflanze von Ophioglossum vulgatum, eine größere Anzahl (bis zehn) Wurzeln und dürften dann fast soviel Jahre als Wurzeln zählen.

Auch bei unserem Gamophyten kann man beobachten, wie ich es für den von $O$. vulgatum zeigte, daß aus dem Erdreich seiner Umgebung einzelne dunkelbraune verzweigte Pilzfäden an ihn herantreten, an seiner Oberfläche fortkriechen und Zweige in sein Inneres abgeben, aber auch durch die Rhizoide ihren Eingang nehmen (Fig. $27 h$ ).

So zeigt sich schon die äußere Gestalt unseres Prothalliums im Vergleiche mit dem von B. virginianum nur als eine kleine Form desselben, des Typus Botrychium, als ein dorsiventrales, elliptisches, auch

1) S. 658 . 
herzförmiges Knöllchen, die Geschlechtsorgane auf der Oberseite tragend, welches den stielrunden Formen des Ophioglossum- und HelminthostachysTypus mit radiärem Aufbau ihres Körpers gegenüber steht.

Dèn Bau unseres Gamophyten sollen uns zunächst die Figuren 28-31 darlegen, welche zeigen, daß sein ei- oder herzförmiger Körper von seinem ältesten hinteren Teile, dem Sporenpol, bis zu seiner meristematischen Region, dem Scheitelpol, aus lückenlos aneinandergrenzenden parenchymatischen Zellen besteht.

Von dem Prothallium-Anfang des B. virginianum gibt uns Jeffrey keine Auskunft. Die Entwicklungsgeschichte des Gamophyten wird uns darüber am besten unterrichten. Diese aber zu liefern will nicht gelingen. Ich erzielte zwar die ersten Entwicklungsstadien der Sporenkeimung unserer Art, welche zunächst Campbells Angaben 1) von B. virginianum bestätigen. Da ich noch weitere Entwicklungszustände erhoffe, so will ich die' Bekanntgebung hierüber zunächst noch hinausschieben.

Wir haben bei der Ausbildung der Farnprothallien sowie bei den Moosen nach Lampa und Jakowatz ${ }^{2}$ ) zwei Entwicklungsstadien zu unterscheiden, nämlich das fadenförmige Anfangs- oder Protonemastadium mit begrenztem Wachstum, an welchem dann als seitliche Anlage das eigentliche Prothallium entsteht. Wir haben also zunächst nach dem Protonemastadium zu fragen. Ein vollständiger Gamophyt unserer Art zeigt an seinem Sporenpol nur eine einzelne Zelle, an der wie an einem Stielchenrudiment das Prothallium haftet (Fig. 27 u. 28a). Dieser in einer Zelle zugespitzte hintere Abschluß des Prothalliums findet sich selten in dessen Median vor, sondern ist meist seitlich verschoben, aber fast immer erhalten. Diese in Fig. 27 u. 28 mit $a$ bezeichnete älteste Zelle vertritt das Protonemastadium, das hier in so rudimentärer Form vorkommt, daß sogar in dieser Zelle selbst die Anlage des ersten Rhizoids fehlt, dessen rudimentäre Form in Gestalt einer kleinen linsenförmigen Zelle uns Belajeff ${ }^{3}$ ) an der Basis der männlichen Prothallien der Wasserfarne kennen lehrte.

Der an die erste Zelle des Prothalliums zunächst anschließende Körperteil besitzt keine Geschlechtsorgane. Sein Inneres besteht aus

1) Campbell, pag. 224.

2) Lampa, Über die Entw. einiger Farnprothallien u. Jakowatz, Vergleichende Unters. über Farnproth. (Sitzungsberichte d. Akad. d. Wiss., Wien 1901, pag. 95 u. 479.)

3) Belajeff, Über die männlichen Prothallien der Wasserfarne, Bot. Zeit., I. Abt., 1898. 
polyedrischen Zellen, die nach der Peripherie hin flacher erscheinen und mit einer Verdickung der äußeren Zellwände abschließen. Seine Zellen sind von einem Pilze bewohnt, der anfangs auch die äußeren Zellen befallen hat, dann, weiter vom hinteren Ende entfernt, diese freiläßt. Somit erscheint der erste Körperteil des Prothalliums radiär gebaut und erhält erst von da ab ein dorsiventrales Gepräge, wo auf seiner Oberfläche die Geschlechtsorgane auftreten.

In diesem Teile finden sich die großen polygonalen, mit Pilzklumpen vollgestopften Zellen in der Mitte und der Bauchpartie des Prothalliums vor, während die den Geschlechtsorganen benachbarten und sie umgebenden Zellen kleiner, auch gestreckter und arm an Inhalt sind (Fig. 28, 29 u. 30). Die äußeren Zellen haben auch hier abgeflachte Form, sie erscheinen inhaltsarm, pilzfrei und schließen nach außen mit verdickten Zellwänden ab. Diese sind aber farblos und nicht lichtbraun, wie Hofmeister angibt. Braun gefärbt sind hier lediglich die basalen Wandteile der Wurzelhaare, mit welchen unser Gamophyt allseitig, wenn auch nicht reich besetzt ist (Fig. 27). Diese Rhizoide entstehen an der wachsenden Region, an der einzelne Zellen der Oberfläche sich über diese ausstülpen und diese Hervortreibung periklin durch eine Wand abteilen (Fig. 28 u. 29), worauf das einzellige Haar durch gutes Spitzenwachstum für seine Aufgabe ergiebig verlängert wird. Seine nach dem Prothalliumkörper abgrenzende Wand sowie sein basaler zylindrischer Wandteil färben sich frühzeitig braun, und letzterer verdickt sich so stark wie die äußeren Zellwände. Nach hinten zu findet man sie abgestorben vor, und der Endophyt nimmt durch sie gern Eintritt in das Prothallium (Fig. 28h). Neben diesen langen einzelligen Rhizoiden entstehen auf der Oberseite während der Entwicklung der Geschlechtsorgane noch vereinzelt kurze einzellige, auch durch quere Wände in mehrere Zellen geteilte Paraphysen (Fig. 28, 29 u. $30 p$ ). Sie sind enger und dünnwandiger wie die langen einzelligen Rhizoide, aber auch an ihrer Basis braun.

Der mediane Längsschnitt der Figur 28 durch eine jüngere Prothalliumform führt von der. äußersten Zelle des Sporenpols ausgehend die mediane Rückenlinie aufsteigend über den radiär gebauten hinteren Teil des Gamophyten an das älteste Antheridium und von da ab auf seinen dorsiventralen Teil, nämlich über eine in verschiedenen Entwicklungsstufen anzutreffende Antheridienreihe und über das jüngste Antheridium abwärts auf die vordere wachsende Region, den Scheitelpol (Fig. 27 u. 28 s). Dieses Scheitelmeristem, welches das Wachstum des Gamophyten einleitet, ist von geringer Ausdehnung und findet sich 
bei jungen Individuen nach -vorn gerichtet und vertieft gelegt vor. Bei dem Vordringen des Prothalliums in das Erdreich wird es geschützt einmal durch die vorwachsenden jüngsten Antheridien der Rückenseite und ferner durch die sich vorschiebenden nächsten Zellelemente der Seiten- und Bauchpartie (Fig. 28s). Bei alten Prothallienformen erscheint das Meristem vorn mehr nach oben gerichtet (Fig. $29 \mathrm{~m}$ ). Durch ein energisches Wachsen der jüngsten, namentlich nach der Bauchseite hin abgeteilten Zellelemente, welche keilförmig gegen das Erdreịch vorschieben, werden hier die empfindlichen Meristemelemente vor Druck des Erdreichs bewahrt.

Das Meristem zeigt im Schnitte zartwandige engere prismatische Zellen, die mit ihrer längsten Ausdehnung senkrecht zur äußeren Fläche gerichtet und durch besondere Ausrüstung mit Baustoffen kenntlich sind (Fig. $28 s$ u. $29 \mathrm{~m}$ ). Ihre geringe Zahl ist leicht $\mathrm{zu}$ übersehen und zu durchmustern. Keine dieser Zellen aber läßt sich als eine nach dem Inneren hin zugespitzte Scheitelzelle erkennen. Auch die Scheitelansicht des Prothalliums von oben aufgenommen (Fig. $31 \mathrm{~m}$ ) zeigt keine durch Form und Teilungsweise gekennzeichnete Scheitelzelle. Wir haben hier also ein Wachstum mit prismatischen Initialen, von welchen eine oder auch mehrere, die gerade das Wachstumszentrum einnehmen, durch anti- und perikline Teilungen die Bauelemente für das Äußere und Innere des Gamophyten erzeugen. Diese Teilungen dürften am Anfang der Vegetationsperiode etwas rege, sonst nur langsam erfolgen. Die Scheitelaufnahme der Fig. 31 läßt wenig, kaum mehr als sechs Zellen des embryonalen Gewebes mit dichterem Inhalt als zum Meristeme gehörig erkennen.

Bei Botrychium virginianum hält Jeffrey das Wachstum des Prothalliums zwar durch eine Scheitelzelle für wahrscheinlich und bildet auch eine solche $a b$, doch war er nicht imstande, ihre horizontale Gestaltung zu finden und zu beschreiben; mithin dürfte bei dem Prothallium von B. virginianum die Wachstumsweise noch nicht endgültig richtig erkannt sein.

Die Wurzelhaare entstehen am fortwachsenden Ende des Prothalliums aus einzelnen peripherischen Zellen immer aufs neue in akropetaler Folge (Fig. 31 rhi). Aus einer solchen Zelle, die ein Wurzelhaar erzeugt, stülpt sich die Mitte der Außenwand hervor, und diese Ausstülpung trennt sich meist durch eine, seltener durch zwei Querwände von der Mutterzelle ab, nimmt durch ein fortdauerndes Spitzenwachstum immer größere Länge und schlauchförmige Gestalt an. Die das Rhizoid von der Epidermiszelle abgrenzende Wand wird frühzeitig braun gefärbt, 
auch die Basis der zylindrischen Wand erhält diese Farbe, dazu verdickte Membran. Nur an dem jugendlichen Teile des Prothalliums sind die Rhizoide funktionsfähig und für das trockene Substrat sehr wichtig. Sicher vermögen sie nicht nur für das Wasserbedürfnis, sondern auch für die vollständige Ernährung zu sorgen. Jedenfalls vermögen diese Rhizoïde die Humusbestandteile des Bodens aufzulösen, aufzusaugen und so die Ernährung zu vermitteln. Der braune Farbstoff macht sicher den basalen Teil des Rhizoids korkartig, so daß zwar die Imbibitionsfähigkeit vermindert, dafür aber die Diffusion des einmal aufgenommenen Wassers in der heißen Zeit erschwert wird. Auch die äußeren Wände zerstörter Zellen werden, wie auch beim Prothallium von Ophioglossum vulgatum, zum Schutze verkorkt. Weiter zurück auf dem mittleren und hinteren Teile unseres Gamophyten scheinen die Wurzelhaare funktionslos zu sein, der von ihnen bedeckte Körperteil ist fertig gebildet, und in seinen Zellen sind Reservestoffe aufgespeichert.

Nur die meristematischen Zellen und die ihnen angrenzenden inneren Zellen, sowie die den Geschlechtsorganen angrenzenden Zellen besitzen neben den plasmatischen Stoffen auch Stärke. Dem großen Teile des Prothalliums aber, in welchem der endophytische Pilz Herberge gefunden hat, fehlt sie. Dafür ist derselbe reich an Öl, welches in großen Mengen in den Ausstülpungen des Mycels des endophytischen Pilzes eingeschlossen wird und sich durch Osmiumsäure schwarz färbt. Aber auch Eiweißstoffe fehlen nicht.

Die größeren und kleineren Klumpen eines halbdurchsichtigen Körpers, mit denen die Zellen unseres Gamophyten vollgestopft erscheinen und die durch Jod nicht gebläut werden, hat Hofmeister ${ }^{1}$ ) nicht übersehen, nur wußte er sie nicht als die Gebilde eines endophytischen Pilzes zu deuten.

Dieser Endophyt ist in jedem Prothallium gegenwärtig. Er bewohnt das ganze Innere des radiär gebauten älteren Teiles und nimmt hier auch am hinteren Ende anfangs von den peripherischen Zellen Besitz, bleibt dann aber jm weiteren den Oberflächenschichten fern (Fig. $28 \mathrm{en}$ ). In dem dorsiventralen Teile befällt er nicht das Rückenzellgewebe, also nicht die Zellen der Geschlechtsorgane und deren Umgebung (Fig. 28 u. 30). Er beschränkt sich hier auf die mittleren und unteren Zellpartien und dringt von hier bis nahe an das Meristem vor (Fig. 28 u. 29). Ihren Eintritt nehmen die braunen Hyphen des Endophyten vornehmlich durch ältere Wurzelhaare, doch vermögen sie

1) Hofmeister pag. 658 
auch durch die kutikularisierte Oberflächenschicht des Gamophyten direkten Eingang zu finden (Fig. 28h). Die braune Hyphe entartet nach ihrem Eintritte in das Prothallium und füllt die Zellen mit ihren Knäueln und größeren oder kleineren sackartigen Hyphenerweiterungen an, ohne den Zellkern zu töten (Fig. 32). Die Stärke schwindet aus dem Inhalte der befallenen Zellen, aber in den darin befindlichen Pilzhyphen werden namentlich Öl und auch Eiðeiß als gute Reservestoffe für eine Entwicklung des Embryos aufgespeichert. So zeigt der endophytische Pilz unseres Gamophyten nach Verteilung und Struktur die gleichen Eigenschaften wie der Endophyt von B. virginianum. Mit dem gleichen Pilz dürften aber auch die Prothallien von Ophioglossum vulgatum und die der übrigen Ophioglossazeen in Symbiose leben.

Der physiologische Nutzen des Zusammenlebens von Prothallium und Pilz scheint mir auch hier nicht darin zu bestehen, daß die wenigen und unscheinbaren Pilzfäden, welche aus dem Substrat des Gamophyten in ihn einmünden, belanglose Humusstoffe ihm zuführen, sondern nur darin, då die von den funktionsfähigen Rhizoiden der wachsenden Scheitelpartie aufgelösten und herbëigéführten Baustoffe mittelst des Stoffwechsels des Endophyten in haltbare Reservestoffe umgewandelt und aufgespeichert werden, von denen namentlich die auffallend große Ölmenge dem Prothallium die Fähigkeit verleiht, in dem sandigen Boden auch während der Sommerhitze und der Winterkälte vor Austrocknung bewahrt zu bleiben.

Die Sexualorgane unseres Prothalliums sind von Hofmeister selbst in ihrer Stellung nicht genügend erkannt. Auch bleibt er jede nähere Angabe über ihren Bau schuldig und hat ihre Entwicklungsgeschichte nicht festgestellt. Durch Jeffrey aber haben wir diese Organe von B. virginianum in Wort und Bild zuerst ausführlich kennen gelernt, und man kann schon im voraus erwarten, daß dieselben auch mit denen unseres Gamophyten übereinstimmen werden.

Auch hier entstehen sie in akropetaler Folge am Scheitelmeristem auf der Oberseite des Prothalliums (Fig. 27 u. 31).

Die Antheridien, welche die Mitte der Rückenseite einnehmen, béginnen ihre Entwicklung am Meristem schon unmittelbar hinter den Scheitelinitialen in der für alle Ophioglossazeen ${ }^{1}$ ) festgestellten Weise aus etner Oberflächenzelle (Fig. 29an), die sich durch ein größeres

1) Jeffrey, pag. 13, ferner: Lang, On the Prothalli of Ophioglossum pendulum and Helminthostachys zeylanica. Annals of botany 1902, Bd. XVI, No. 61, und Bruchmann, Über das Prothallium und die Keimpflanze von Ophioglossum vulgatum L. Bot. Zeit. 1904. 
Volumen, dichteres Plasma und größeren Zellkern von den benachbarten Zellen unterscheidet und zuerst durch eine quere Wand teilt. Aus der inneren Zelle entwickelt sich durch wiederholte Teilungen die große Anzahl von Spermatozoidenmutterzellen. Mit dem Alter nehmen die Antheridien an Größe zu und treiben meist den Rücken des Prothalliums auf (Fig. 28 u. 30 an).

Die Deckzelle, welche durch die starke Vermehrung der unter ihr liegenden Zellen veranlaßt wird sich $\mathrm{zu}$ dehnen und $\mathrm{zu}$ teilen, gibt der Deckschicht den Ursprung. Sie läßt, von oben gesehen, ihre Teilungen mit senkrecht zu einander gerichteten Wänden ausführen (Fig. 31 an), und solche Teilungen bringen nicht immer eine, sondern, was hier besonders hervorzuheben, zwei, auch drei Öffnungszellen (Fig. 28 u. 33). Die Deckschicht des Antheridiums wird bis auf die Öffnungszelle zu einem doppelschichtigen Zellager ausgebaut. Bei den Öffnungszellen, als den bei der Ausweitung jüngsten Teilungszellen, dürfte eine Querteilung vielleicht in allen Fällen ausbleiben. Die Antheridien, die meist zu zweien, seltener zu dreien dicht bei einander entstehen können, reifen nur langsam und entleeren sich erst in größerer Entfernung vom Scheitel des Prothalliums (Fig. 28), so daß zu vermuten ist, die in einer Vegetationsperiode entstandenen Organe dieser Art gelangen erst in der nächsten oder übernächsten zur Reife, wenn also die gleichalterigen Archegonien längst verblüht sind oder schon einen Embryo einschließen. So zeigt Fig. 30 einen Querschnitt durch gleichalterige Prothalliumteile, die neben noch unreifen Antheridien schon verblühte Archegonien und einen mehrzelligen Embryo aufweisen. Die reifen Antheridien leeren sich in derselben Weise, wie ich es für Ophioglossum vulgatum feststellte ${ }^{1}$ ). Die Wandzellen sind auch hier durch ihre Quellbarkeit beteiligt, und die entleerten Antheridien zeigen auch hier hervorgewölbte gebräunte Innenwände (Fig. 28), was leider Jeffrey für das Prothallium von $\mathrm{B}$. virginianum nicht hervorhebt.

Die Spermatozoiden haben die bei den Farnen gewöhnliche Gestalt. Sie sind aber hier um etwa die Hälfte kleiner als die von Ophioglossum vulgatum (Fig. 34).

Die Archegonien entstehen in akropetaler Folge am Scheitel des Prothalliums zu beiden Seiten des Antheridienrückens (Fig. 31 ar). Sie entwickeln sich sehnell, erlangen noch in derselben Vegetationsperiode Geschlechtsreife und verblühen, bevor noch die gleichalterigen Antheridien vollständig entwickelt sind. Ihre Entwicklung stimmt mit

1) Bruchmann, Bot. Zeit. 1904. 
denen der anderen Ophioglossumarten überein. Sie unterscheidet sich nicht von meiner Darlegung des Archegoniums von O. vulgatum. Auch hier finde ich nicht, daß eine Bauchkanalzelle abgegliedert wird, die aber Jeffrey bei B. virginianum deutlich gebildet vorfand. Daß die Archegonien dem Prothallium nicht vollständig eingesenkt erscheinen, wie Hofmeister angibt, soll uns die Abbildung eines ausgebildeten Archegoniums kurz vor seiner Öffnung zeigen, welches mit seiner oberen Halspartie über die Prothalliumoberfläche hervorragt: die abgestorbenen Archegonien verengen ihren Kanal und verkorken in ihm die äußeren Zellwände.

So erscheint denn unser Gamophyt sowohl nach seiner äußeren wie auch nach seiner inneren Gestalt als ein guter Repräsentant des Botrychium-Typus, der keineswegs mit dem Prothallium von B. virginianum in Gegensatz gestellt werden kann. Wir haben also einen dicken Strich durch Hofmeisters Darstellung desselben auf Tafel XII zu machen, so durch die in viele Lehrbücher aufgenommene Fig. 1, den medianen Längsschnitt, der neben Antheridien auch ein Archegonium auf der Oberseite zeigt, dann ferner auch durch den von Fig. $6 b$, welche ein Prothallium mit Antheridien auf der Oberseite und Archegonien auf der Bauchseite darstellt u. a. m. Bedauerlich ist nur, daß solche Nachprüfung der Hofmeisterschen Darstellung nicht schon längst geschehen und daß auch ferner die Entwicklungsgeschichte dieses Prothalliums noch nicht erforscht werden konnte.

\section{Der Sporophyt.}

a) Von der älteren Pflanze.

Die Keimpflanze zeigt hier, wie auch bei Ophioglossum vulgatum, anfangs ein mehrjähriges, nur unterirdisches Wachstum, bei welchem sie zwar kräftige Wurzeln erzeugt, aber in der Entwicklung ihres Sproßteiles auffallend zurückhält und nur eine Reihe unvollkommener Blätter, nach Hofmeister „niederblattartige Wedel" hervorbringt. Einen klaren Einblick in diese ersten Entwicklungen können uns nur die Studien der Sproßteile der älteren Pflanze bringen.

Die Gipfelknospe der Ophioglossazeen mit den eigenartigen Blattorganen, die ihresgleichen nicht weiter in der Farnwelt haben, wird immer aufs neue ein anziehendes Objekt des Studiums abgeben. Um ihre Klarlegung haben sich schon eine Reihe Forscher bemüht, so z. B. Al. Braun, Roeper, Hofmeister und namentlich Holle ${ }^{1}$ ). Uns soll

1) Holle, Über Bau und Entwicklung der Vegetationsorgane der Ophioglossazeen. Bot. Zeit. 1875, pag. 241 u. f., wo auch die Literatur einzusehen. 
hier nur die Gipfelknospe von B. Lunaria wegen unseres Interesses für ihre Keimpflanze beschäftigen.

Eine am Ende ihrer Vegetationsperiode (im Juli) ausgegrabene ältere Pflanze unseres Zwergfarns mit ihrem kurzen, aufrechten Rhizom und den von diesem wagrecht ausstrahlenden starken braunen, auch öfter verzweigten Wurzeln mißt mit ihrem einzigen oberirdischen Doppelblatt kaum ein Spanne, und doch hatte das Blatt zu seiner Ausbildung, wie bekannt, 5 Jahre nötig, wovon es 4 Jahre unter dem Boden zubrachte.' Dieses ausgebildete Blatt gliedert sich in die beiden gefiederten Teile der einander zugekehrten fertilen und sterilen Lamina, dem Stiele und der Scheide, von welcher die übrigen Teile der Gipfelknospe völlig eingeschlossen sind. Entfernt man das ausgewachsene Blatt, so tritt uns aus dessen Scheide das für das nächste Jahr zur oberirdischen Entfaltung bestimmte Blatt mit seinen fast fertig angelegten Teilen in schwach grünlicher Farbe entgegen (Fig. 51). Trennt man nun die ganze Gipfelknospe von dem Rhizom der Pflanze über der jüngsten Wurzel ab und verkürzt dieselbe von unten her durch Querschnitte, bis sich die Scheiden der ineinander geschachtelten Blattanlagen erkennen lassen, so können diese leicht freigelegt werden (Fig. 51-54). Von dem innersten Blatte aber dürfte nur seine abgeschnittene stumpfkonische Kuppe gewonnen sein; der basale Teil findet sich dann noch auf unserem letzten Stammquerschnitt vor, wo er dessen Scheitel deckt. Befreit man den Scheitel von solcher Decke, so gelangt man zur Erkenntnis des allerjüngsten letztjährigen Blatthöckers und der Ausdehnung der flachen freien Scheitelfläche. Somit haben wir in der Gipfelknospe fünf Blattformen im Alter von $1-5$ Jahren zu unterscheiden, bei denén jedes jüngere Blatt in dem nächst älteren völlig eingeschlossen erscheint und die in einer Schraubenstellung, bei einigen Pflanzen in Zeiger-, bei anderen in Gegenzeigerrichtung aufgebaut werden.

Diese ganz schnell vorzunehmende Orientierung über die Zahl, das Alter und́ die Stellung der Blätter der Gipfelknospe hat uns aber die Einsicht auf die Blattbasen, die durch die Querschnitte abgetrennt wurden, entzogen. Entfernt man von der von dem Rhizom abgetrennten Gipfelknospe das fünf- und das vierjährige Blatt, so daß das dreijährige frei den Scheitel glockenartig umschließend gewonnen wird (Fig. $52 b$, Fig. 53), so erkennt man an seinem Grunde unterhalb seines fertilen Teiles einen Querspalt, der im späteren Alter verwächst, am zweijährigen aber, wie auch am dreijährigen nicht zu übersehen ist. Schon Röper entdeckte diese Blattspalte, die Hofmeister zuerst richtig deutete und 
auch Holle ausführlich behandelt ${ }^{1}$ ). Sie ist ein mundartiger Verschluß, dessen Oberlippe und Mundwinkel von dem vorderen Rande des den Scheitel umwachsenden und auf den Stammgrund deckelartig aufgelegten Blattes gebildet werden.

Wir übersehen nun noch einmal diese interessanten Gebilde der Gipfelknospe an der schematischen Darstellung ihres Längsschnittes, der die in verschiedenen Ebenen liegenden Blattmedianen der besseren Übersichtlichkeit wegen in gleicher Ebene einander gegenüberstellt (Fig. 59). Wir finden den engen Scheitel $(s)$ und die geringe Erhöhung des einjährigen $\left(b_{1}\right)$ Blattes, das zweijährige $\left(b_{2}\right)$ in Form eines stumpf konischen Deckels. Es hat seine Verschlußspalte wie die älteren Blätter an der seiner Insertionsstelle entgegengesetzten Seite und steckt ganz in der Blattscheide des nächsten um ein Jahr älteren Blattes $\left(b_{3}\right)$. Letzteres zeigt an seinem Gipfel die in Entwicklung begriffenen, einander gegenübergestellten beiden Blattlamina, die im vierten Jahre gestielt und völlig ausgebildet erscheinen. Während die Hohlräume der Scheiden den umschließenden Blättern nicht größeren Raum geben, als deren Gestalt benötigt, hat das vierjährige Blatt $\left(b_{4}\right)$ in der gestreckten Scheide des sich über der Erdoberfläche entfaltenden fünfjährigen freieren Spielraum und durchbricht endlich das dann schon abgestorbene Gewebe derselben im nächsten Jahre. Es entfaltet sich im Frühjahre seines fünften Entwicklungsjahres im Lichte, assimiliert, bringt die Sporen zur Reife und stirbt schon vor Beginn des Hochsommers ab. Somit gewinnt es also nach so langer vorbereitender Entwicklungszeit nur eine kurze Arbeitszeit.

Die freie Scheiteloberfläche des Rhizoms ist meist flach, auch vertieft und von geringer Ausdehnung (Fig. 55). Sie zeigt, wie bekannt, ein Wachstum mit einer dreiseitigen pyramidenförmigen Scheitelzelle, die oft sehr zusammengedrückt erscheint und dann im Längsschnitt tiefe und enge Segmente aufweist (Fig. 57 a). Ihre regelmäßigen Teilungen dürften, entsprechend der langsamen Erhebung der Spitze des Rhizoms. nur langsam aufeinanderfolgen, und wenn jedes Segment einem Blatte den Ursprung gäbe, würde auch jährlich ein Segment vollständig ausreichend sein und nach einem Umlauf der Segmentierung der Scheitelzelle erst im vierten Jahre an derselben Seite die Wiederholung der Teilung eintreten. Die erste quere Teilungswand der Segmente _im Längsschnitte gesehen legt auch hier die von Campbell ${ }^{2}$ ) ausgesprochene Vermutung nahe, daß sie die Scheidewand zwischen

1) Holle, Bot. Zeit. 1875, pag. 272.

2) Campbell, pag. 244 . 
Plerom und Rinde sei. Allein bei solchem so wenig hervortretenden Scheitelwachstum des Rhizoms sind weder diese noch die weiteren Teilungen in Rinde und Epidermis klar zu erkennen.

Das junge Botrychium-Blatt soll sich nach Holle ${ }^{1}$ ) als eine ordnungslose Zellgruppe am Scheitel des Stammes erheben und sein Wachstum soll weder eine einzelne Zelle, noch ein Zellenkomplex beherrschen. Er belegt diese Ansicht durch Abbildungen meist von B. rutaefolium „mit möglichst genauer Wiedergabe der einzelnen Zellen" und zeigt, daß an eine Scheitelzelle hier nicht $\mathrm{zu}$ denken ist. Dagegen gibt Campbell²) von B. virginianum an, daß das junge Blatt anfänglich eine Scheitelzelle besitze, die in ihrem Wachstum mit der des Stammes übereinzustimmen scheine. Die den Text begleitende Abbildung eines jungen Blattes veranschaulicht sie aber nicht (siehe Fig. 124). Somit macht sich bei solchen Widersprüchen eine eingehende Untersuchung der Blattanlage und ihres Wachstums bei unserer Art nötig.

Recht junge Höcker einer Blattanlage am Scheitel der Rhizome zeigen uns die Pflanzen im Frühjahre, beim Beginne ihrer Vegetationsperiode. Solch eine sich eben erhebende Zellgruppe der jungen Blattanlage (Fig. $\check{5} 5 b_{1}$ ) ist nicht ordnungslos, sondern sie besitzt zwei Merkmale, die dagegen zeugen. Einmal erhebt sie sich stets in einer geraden Frontlinie an der der freien Scheitelfäche zugekehrten Seite über dieselbe. Die in Fig. 55 dickere, in gerader Richtung gezeichnete Linie zeigt die verdickten Zellwände der sich eben über die Scheitelniveaugrenze erhebenden Protuberanz. Den höchsten Punkt derselben nimmt eine nicht zu verkennende dreiseitig-pyramidale Scheitelzelle ein (Fig. 55 b), deren angrenzende Segmente die bekannte gesetzmäßige Abgliederung bekunden. Die Scheitelzelle und ihre nächsten Segmente, die erstere oft an Größe übertreffen, besitzen größere Zellkerne und dichteren plasmatischen Inhalt als die diesen angrenzenden Zellen, und es sind diese fünf Zellen der Blatthöckermitte, von denen die mit $b_{1}$ bezeichnete Zelle die Scheitelzelle darstellt, unstreitig aus einer einzigen Zellinitiale, die in der Mitte einer älteren Segmentreihe der Rhizomscheitelzelle lag, entstanden. Die ihnen seitlich und im Rücken angrenzenden Zellen beteiligen sich auch an der Erhebung. Dadurch nun, daß der junge Blatthöcker in gerader Frontlinie über den Scheitel emporsteigt, dürfte er bekunden, daß er wahrscheinlich einem einzelnen Segment der Stammscheitelzelle zugehört, und zwar nicht dem letzten, sondern dem, das im letzten Turnus der Segmentreihe nach solcher Stammseite abge-

1) Holle, Bot. Zeit., pag. 272.

2) Campbell, pag. 244 . 
schieden war. Wenn der junge Blatthöcker eine nicht mehr zu verkennende Form angenommen hat, findet sich in der noch flachen Scheitelpartie in der Mitte einer Segmentreihe eine größere, auch mit dichterem Inhalt ausgestattete Zelle, die auch eine schiefe Teilung eingeht (Fig. $56 b_{1}$ ). Diese Zelle dürfte als die Initiale des im nächsten Jahre zu bildenden Blatthöckers anzusprechen und ihre einleitende Teilung als allererster erkennbarer Anfang einer Blattbildung anzusehen sein. Auch im medianen Längsschnitt wird die Scheitelzelle der jungen Blattanlage nebst ihrer Teilungsweise nicht übersehen werden können (Fig. $57 b_{1}$ ). Sie liegt stets auf dem gewölbten oberen Teile, nicht am Vorderrande, an dem die Blattzellen in einem scharfen Winkel über den flachen Stammscheitel sich erheben. Gleich nach dem Hervortreten der jungen Blattanlage macht sich ein doppeltes Wachstum derselben bemerkbar. Einmal tritt an der Front der Blattinsertion ein von dieser abstrebendes energisches Randwachstum auf, welches die Anlage stark nach vorn ausdehnt und sie keilig über den Rhizomscheitel unter dem nächstältesten Blatte vorschiebt (Fig. 56). Dann zeigt sich das aufstrebende Scheitelwachstum, vermittelt durch die dem gewölbten Blattrücken aufsitzende Scheitelzelle. Diese ist nicht von besonders hervortretender Größe. Ihre Teilungen scheinen nur langsam aufeinander zu folgen. Doch wachsen und teilen sich ihre Segmente lebhaft, und zwar die der Rückenseite stärker, so daß auch durch das Scheitelwachstum die Ausbreitung der jungen Blattanlage über den Stammscheitel unterstützt wird.

Im ersten Jahre bringt es die Blattanlage nur zu einem geringen, sich über den Stammscheitel vorschiebenden Zellkörper; erst in der zweiten Wachstumsperiode wird das Überwachsen des Stammscheitels vollendet und die Ausbildung einer eigenartigen stumpfkonischen Deckelform erzielt, die überall dicht, ohne Hohlräume zu bilden, anschließt und sich hinter dem Rücken der einjährigen Blattanlage auf die Stammoberfläche anlegt, auch hier die obenerwähnte Querfurche bildet (Fig. 57). Dieser eigenartige Blattkörper von Botrychium ist gleichsam eine vom Grunde auf gebildete Verwachsung von Scheide und Blatt, die ja bei Ophioglossum dem Rhizomscheitel getrennt entspringen.

Im Längsschnitt sehen wir das junge Blatt über dem Prokambium des Rhizoms entstehen und dieses bei dem zweijährigen Blatt direkt aus dem Stamme als Prokambiumstrang nach dem Gipfel des Blattmeristems geführt (Fig. $57 b_{2}$ ). Bis dahin ist innerhalb zweier Jahre wenig mehr als der Teil entwickelt, der den basalen, den Scheidenteil des ausgebildeten Blattes ausmacht. 
Noch Ende des zweiten oder Anfang des dritten Jahres wird in der Blattentwicklung zur Anlage der eigenartigen einander gegenübergestellten sterilen und fertilen Blattlamina geschritten. Ähnlich wie bei einer Knospung treten auf dem konischen Scheitel durch seine Teilung die beiden in der Mediane des Blattes stehenden Höcker hervor, von denen jeder seine dreiseitige Scheitelzelle am Vorderrande des Blattgipfels trägt (Fig. $57 b_{2}$ und Fig. $58 b$ ). Die Untersuchung über ihre erste Anlage konnte ich nicht ganz zum Abschluß bringen. Ich fand bei der Anlage dieser Gipfelteilung die beiden Scheitelzellen nahe beieinander und vermute, daß, während die ursprüngliche Gipfelzelle für die sterile Lamina beibehalten wird, aus einem nach abwärts angrenzenden Segmente die der fertilen Lamina ausgebildet wird (Fig. $57 b_{2}$ ). Bemerken aber will ich noch, daß vielleicht auch, da doch zwei ganz neue dorsiventrale Blattgebilde angelegt werden, die Auflösung der alten Scheitelzelle und dafür eine Hervorbildung zweier neuer nicht unmöglich ist.

Das sterile Blatt, welches die direkte Fortsetzung der ursprünglichen Wachstumsrichtung wird, ist anfangs das kräftigere und vorgeschrittenere, hinter dem das fertile zurückbleibt (Fig. 58). Bei jungen Pflanzen kann sich letzteres auch nur auf die Anlage beschränken. Solche Fälle beobachtete Hofmeister und kam dadurch zu der Ansicht, daß erst die fertile Lamina angelegt werde, wenn die sterile ihre Fiedern zu entwickeln beginne.

Im dritten Entwicklungsjahre der Blätter sind ihre Scheitelzellen noch immer in Tätigkeit (Fig. 58 b), auch wenn schon in akropetaler Folge durch das Randwachstum am unteren Teile die Blattfiedern entstehen. Erst wenn dieses Randwachstum auch die Gipfel der beiden Lamina erfaßt, erlischt endlich das Scheitelwachstum mit der dreiseitigen Scheitelzelle, was bei fortgeschritteneren Blättern bereits am Ende des dritten, bei anderen aber erst im vierten Jahre stattfindet.

Mit dieser Studie über die Blattknospe von $\mathrm{B}$. Lunaria begnügen wir uns zunächst. Über die Ausbildung der Fiedern beider Blattarten, über die Sporenentwicklung, sowie über den Gefäßbündelverlauf, welcher namentlich durch seine radiale Anordnung in den Stielen der Blätter bemerkenswert erscheint, geben unten genannte Autoren nähere Auskunft ${ }^{1}$ ).

1) Goebel, Beiträge zur vergleichenden Entwicklungsgeschichte der Sporangien. Bot. Zeit. 1880 u. 1881. Organographie der Pflanzen, p. 758.

Holzmann, On the apical growth of the stem and the development of the sporangium of Botrychium. Bot. Gazette 1892.

Bower, Studies in the Morphology of spore-producing members. II. Ophioglossaceae. London 1891.

Campbell, Mosses u. Ferns, 1895, pag. 250. 
Wir sind nun genügend vorbereitet, um über die Verhältnisse, welche uns die Keimpflanze in der verzögerten Entwicklung ihrer Stammknospe darbietet, ein richtiges Urteil zu gewinnen.

b) Von der Keimpflanze.

Das Prothallium von Ophioglossum vulgatum gab zu erkennen, daß eine Befruchtung der Archegonien sehr selten eintrete, ich fand nur wenige Embryonen und Keimpflanzen vor. Bei den Prothallien von B. Lunaria dagegen besitzt fast jedes größere Exemplar einen Embryo (Fig. 19-21 em), einige sogar deren mehrere (Fig. 24 u. 25) und selbst, wenn eine größere Keimpflanze aus dem Prothallium hervorgewachsen war, konnten die Archegonien seines weiterwachsenden Teiles noch Befruchtung finden. Aber mehr als zwei Keimpflanzen dürften von dem verhältnismäßig kleinen Gamophyten nicht zur vollständigen Ausbildung gebracht werden können. Ich traf kein Prothallium mit drei entwickelten Sporophyten an. Dabei wachsen die Keimpflanzen über Erwarten schnell hervor. So z. B. gewann ich von einem Prothallium in meiner Kultur mit einem Embryo von der Größe der Figur $19(\mathrm{em})$ nach vier Wochen eine Keimpflanze von der Größe der Figur 22. Aber, wie Hofmeister, fanid auch ich eine Anzahl junger Keimpflanzen, die durch ein Absterben ihres Mutterprothalliums frühzeitig selbständig geworden sind. Es waren dies kleine, braune, länglich-eiförmige Gebilde von einer Entwicklungsform, wie sie durch die Figuren 7,8 und 26 gekennzeichnet sind.

Von der Entwicklung der Keimpflanze soll nun folgendes berichtet werden. Das befruchtete Archegonium schließt seinen Halskanal durch Zusammenziehen der Halszellen (Fig. 36), und die Eizelle vergrößert sich etwas, bevor sie ihre erste Teilung eingeht. Sie teilt sich zuerst quer zur Achse des Archegoniums in zwei gleiche Teile (Fig. 37), dann rechtwinklig zu dieser Wand in vier (Fig. 38) und weiter durch eine senkrecht auf die ersten beiden Wände gerichtete Wand in acht gleiche Zellen, wie diese Teilungsweise in Oktanten in derselben Weise für alle Farne und auch für B. virginianum durch Jeffrey nachgewiesen worden ist.

In dem nun folgenden Entwicklungsgang des Embryos nehmen die Teilungen einen unregelmäßigen Verlauf und erzielen zunächst die Ausbildung eines kugeligen oder ellipsoiden Zellkörpers ohne jede erkennbare Gliederung. Wenn auch anfangs in einzelnen Fällen die Basalwand noch als Grenze zwischen der epibasalen und hypobasalen Keimhälfte bemerkbar bleibt (Fig. 39), so geht sie doch im weiteren Wachstum verloren (Fig. 40). Das Gewebe des Mutterarchegoniums 
und der angrenzenden Zellen wird bauchig aufgetrieben und umscheidet den sich vergrößernden Keimling, wie die Prothallien der Figuren 19, 20 und 27 solches darstellen und an Fig. 41, im Schnitte gezeichnet, veranschaulicht ist.

Bei den echten Farnen werden ja die einzelnen Organe der Keimpflanze schon früh voneinander gesondert, und es wird ihre Zurückführung auf die einzelnen Oktanten des Embryos ohne Schwierigkeiten möglich, doch für die Ophioglossazeen bleibt dies ausgeschlossen. Jeffrey glaubt zwar noch für die Keimpflanze von B. virginianum, trotz der späten Differenzierung ihrer Organe, im allgemeinen Sinne sagen zu können, daß aus dem hypobasalen Teile der Fuß und aus dem epibasalen die übrigen Organe differenziert werden. Aber die Keimesentwicklung von B. Lunaria kann eine Stütze für diese Ansicht infolge eines auffallend späten Hervortretens der Organe nicht abgeben. Auch wenn der eiförmige Keimling das Prothallium durchbricht, findet sich zunächst in seinem vorgeschobenen Teile noch nicht die Wurzel angelegt.

Solange der Embryo vom Prothallium umschlossen wird, sind seine Zellen mit dichten plasmatischen Stoffen gleichmäßig erfüllt (Fig. 36-41). Wenn er darauf aber als ovaler Keimling das Prothallium durchbricht, werden in seinen Zellen reiche Mengen Stärkekörner sichtbar, die äußeren Zellwände seiner freigewordenen Partien verdicken und bräunen sich und die Zellen seines achsilen Teiles erscheinen etwas gestreckt (Fig. 42). Nach dieser Entwicklungsform tritt dann bei unserem Keime endlich die Anlage seiner Organe hervor (Fig. 43).

In dem meist wagerecht von dem Prothallium vorgeschobenen und dem Erdreich zugeführten Teile der jungen Keimpflanze entsteht die dreiseitige Wurzelscheitelzelle und übernimmt die Sorge für die Zellbildung zum Spitzenwachstum der Keimwurzel (Fig. 43 zes).

Der von dem Prothallium noch eingeschlossene Teil, welcher der Wurzel gegenüber liegt, wird als das Saugorgan, der Fuß, erkennbar, der in diesem Entwicklungsstadium und schon vorher ein kräftiges Wachstum durch fleißige perikline und antikline Teilungen aufweist und tiefer in das Prothallium eindringt (Fig. 43f). An diesem der Hauptsache nach aus Wurzel und Fuß bestehenden oviden Zellkörper entsteht seitlich beim Fuße, der Mündung des Archegoniums zugekehrt, die Scheitelzelle des Stammscheitels (Fig. 43s). Man kann immer von großem Glücke sagen, wenn es gelingt, junge Keimpflanzen von solcher Entwicklungsgröße so gut median zu schneiden, daß auch der eben differenzierte Rhizomscheitel zur Anschauung gelangt. Stehen dieselben noch mit dem Prothallium im Zusammenhange, so ist durch dieses eine 
Orientierung gegeben. Bei freien Keimpflanzen aber fehlt solche, da die Scheitelstelle von außen zunächst nicht erkannt werden kann.

Als erste Differenzierung des Stammscheitels tritt hier eine größere peripherische, mit dichten plasmatischen Stoffen ausgestattete Zelle auf, die alsbald von den benachbarten Zellen überwallt und eingeschlossen wird und schon an ihren ersten Teilungen als die erwartete dreiseitige Rhizomscheitelzelle erkannt wird (Fig. 43s, 44s).

Die schützende Überwachsung der jungen Scheitelanlage, namentlich aber die von einer Seite mehr hervorgeschobenen Zellen dürften hier wohl als das sehr rudimentäre Keimblatt gedeutet werden (Fig. 44c). Diese fest verwachsende Umscheidung gibt der Ausweitung der Scheitelstelle durch Ausdehnung seiner Zellen etwas nach, wird aber in der weiteren Entwicklung der Keimpflanze von dem sich bildenden ersten Blatte gesprengt, und letzteres deckt darauf als flache Blattschuppe schützend den Rhizomscheitel.

Wir sehen, daß diese jugendliche Keimpflanze ein ganz anderes Gepräge hat, wie die von B. virginianum, mit ihrer besser hervortretenden Ausbildung der Rhizomknospe. Ähnlich, wie die junge Keimpflanze von Ophioglossum vulgatum, ist auch die unsere der Hauptsache nach ein Wurzelkörper, von dessen Scheitelspitze langgestrecktes prokambiales Gewebe auf die Stammscheitelstelle führt, und die hier nur aus wenigen Zellen, nämlich aus der Scheitelzelle und deren Umschließungszellen besteht (Fig. 43). Bemerkenswert ist noch der Fuß, welcher anfangs wenig hervortritt, dann aber, namentlich wenn die Keimpflanze mit dem Prothallium verbunden bleibt, zu einem auffallend starken halbkugelfö̀rmigen Höcker hervortreibt (Fig. 45f.).

Dieser Fuß schließt am hinteren Ende der ersten Wurzel neben dem versteckten Scheitel die Keimpflanze stumpf keulenförmig ab und ist selbst bei älteren Pflanzen als ein Ansatz an deren erster Wurzel immer deutlich zu erkennen. Sein Inneres besteht aus größeren parenchymatischen Zellen, die an der Peripherie flach sind. Ein rudimentäres Gefäßbündel aber, das Hofmeister vorgefunden haben will, fehlt. Die ungewöhnliche Dicke, zu der hier dies Organ entwickelt und aus dem Prothallium hervorgetrieben wird (Fig. 22-25), läßt erkennen, daß es hier mehr als ein Saugorgan vorstellt. Es dient augenscheinlich gleichzeitig zur Speicherung der dem Prothallium entnommenen Nährstoffe, mit denen die Keimpflanze längere Zeit zweckmäßige Unterstützung findet, und man sieht an älteren Pflanzen, daß die Zellen des Fußes ihren Inhalt verlieren, also an die wachsende Pflanze abgeben, sich bräunen und absterben. 
In solcher Entwicklungsform der Keimpflanze, wie sie durch Fig. 45 dargestellt ist, hat sich schon ihre Verbindung mit dem endophytischen Pilze vollzogen, der aber nicht vom Prothallium aus, sondern von dem Erdreich her an verschiedenen Stellen der Epidermis des Fußes und der Wurzel der Keimpflanze seinen Eintritt genommen hat. Seine Hyphen füllen die Zellen des Fußes und der äußeren Rinde an. Die Epidermis, die Wurzelspitze und ihr Zentralbündel nebst innerster Rinde, sowie die Scheitelknospe und das Rhizomgewebe bleiben pilzfrei. Wie bekannt, verlieren die verpilzten Zellen ihre Stärke, und als Produkte der Umwandlung sind Öl und Eiweiß vorhanden.

Die Mykorrhiza von B. Lunaria und einer Anzahl anderer Arten dieser Gattung ist von Grevilius ${ }^{1}$ ) und neuerdings von Marcuse ${ }^{2}$ ) behandelt worden. Letzterer nimmt mit Stahl an, daß den Kommunikationshyphen solcher Saprophyten eine der physiologischen Funktion der Wurzelhaare ähnliche Aufgabe zu erfüllen zukomme. Zu der Zeit aber, da die Keimpflanze zuerst von dem Pilze befallen wird, ist sie so stark mit Nährstoffen angefüllt, daß sie eine Beihilfe nicht nötig haben dürfte, und dann erzeugt sie eine große Anzahl langgestreckter, auch sich endogen auszweigender Wurzeln, die gewiß auch selbständig den Humus des Bodens auszubeuten imstande sind. Jeffrey findet, daß sich der Endophyt der Sporenpflanze von B. virginianum von dem des Prothalliums unterscheide, eine Ansicht, die ich für unsere Art nicht annehme. Wenn auch der Endophyt in der Sporenpflanze in einigen anderen Eigenschaften entgegentritt als in dem Gamophyten, so können diese auch durch den Charakter des Wirtes bedingt werden.

Wenn kaum durch wenige Teilungen der Stammscheitelzelle das Rhizom begründet ist und eben erst ein kleiner Blatthöcker des ersten Blattes sichtbar wird, schreitet die Keimpflanze schon zur Anlage einer zweiten Wurzel. Die Anlagestelle derselben ist keine bestimmte, sie kann zur Achse der Keimwurzel sehr verschiedene Richtung nehmen, in einem Winkel zu ihr tritt ihre Anlage am häufigsten auf (z. B. Fig. 9, 10, 11 u. 25), aber auch in derselben oder in entgegengesetzter Richtung wächst sie hervor. Zur übersichtlichen Darstellung derselben wurden letztere Fälle gezeichnet (Fig. 45 u. $46 w^{2}$ ). Holle bemerkt, $\mathrm{da} \beta$ er bei seinen Untersuchungen an älteren Pflanzen die erste Anlage einer Wurzel nicht gesehen habe. Bei dem Studium der Keimpflanze

1) Grevilius, Über Mykorrhizen bei der Gattung Botrychium, Flora, Bd. LXXX.

2) Marcuse, Anatomisch-biologischer Beitrag zur Mykorrhizenfrage. Inaugural-Dissertation, Jena 1902. 
hat man öfter Gelegenheit, solche anzutreffen. Sie wird ganz so angelegt wie bei den echten Farnen, indem eine endogene junge Rindenzelle des Stammes sich zur Wurzelscheitelzelle ausbildet (Fig. 63w). Nach ihrer Anlage entsteht eine starke Zellvermehrung der jungen Wurzel in der bekannten Weise, ein Aufquellen der ganzen Scheitelstelle (Fig. 26) und Hervordrängen der Wurzelhaubenschichten, bis endlich die junge Wurzelknospe hervorgewachsen ist und in meist horizontaler Richtung in das Erdreich eindringt. Rückwärts an ihrer Ansatzstelle umfaßt sie schützend die junge Stammknospe und umwächst sie so, daß das Rindengewebe des Stammes vollständig mit dem der Wurzel verwächst, jedoch bleibt die Verwachsungsstelle immer erkennbar (Fig. 46 u. 47). Das junge Rhizom wird gleichsam in einem Wurzelgehäuse eingeschlossén und ragt nicht über das Niveau der letzten Wurzelansatzstelle hervor (Fig. 46). Das Gefäßbündel der zweiten Wurzel wird an das der ersten angesetzt, wie es Fig. 46 veranschaulicht. In gleicher Weise kann nun eine dritte, ja vierte Wurzel am Rhizome entstehen, ohne daß sein Scheitel merklich über seine Wurzelansatzstellen hervortritt. So zeigt Figur 48 eine Keimpflanze mit vier älteren und einer jungen Wurzel, und ihre Stammknospe ist dennoch wenig über die Ansatzstelle der fünften Wurzel emporgestiegen. Hat es dagegen eine Keimpflanze auf sechs bis acht Wurzeln gebracht, so erscheint bei jeder derselben die Rhizomknospe immer merklich hervorgetrieben (Fig. 14-16). Der schematische Durchschnitt einer solchen Keimpflanze, den Fig. 49 darstellt, zeigt den Stammscheitel schon wesentlich aus der Umklammerung der Wurzelansatzstellen hervorgearbeitet. $\mathrm{Da} B$ alle Wurzeln der Keimpflanze von demselben Endophyten bewohnt werden, soll hier noch vorübergehend hervorgehoben werden.

Es ist auffallend, daß unsere Keimpflanze, die von Anfang an in ununterbrochener Folge sich beeilt, eine Reihe kräftiger Wurzeln zu erzeugen, dennoch in der Ausbildung ihrer Stammknospe so zurückhält. Während bei B. virginianum schon gleich das handförmig ausgebildete Keimblatt über die Erdoberfläche treibt, ergrünt und assimiliert, so kommt bei unserer Keimpflanze in gleicher Aufgabe kaum erst das achte bis zehnte Blatt über die Erde hervor.

Wie wir schon gesehen, ist das Keimblatt hier ganz rudimentär und auf wenige Zellen zurückgeführt (Fig. 44c). Der durch eine dreiseitige Scheitelzelle gegründete Stammscheitel wächst langsam und hat in einer Keimpflanze von der Entwicklung der Figuren 26 und 45 noch wenig Bedeutung erlangt, schreitet aber zur Anlage des ersten Blattes. Dieses sowohl wie auch alle übrigen Blattanlagen entstehen 
in der Weise, wie wir sie oben an der älteren Pflanze kennen lernten (Fig. 56). Sie wachsen mit einer dreiseitigen Scheitelzelle auf ihrem Gipfel und durch ein ergiebiges Randwachstum als eine flache Blattschuppe über den Stammscheitel aus, unter deren Schutze sich dann eine neue Blattanlage erheben kann. Durch eine interkalare Ausweitung und Streckung der jungen Stammknospe wird die primitive Kotyledonarscheide durch die erste Blattanlage frühzeitig gesprengt, und das erste Blatt gelangt mit seiner erhabenen Seite als eine unvollständig ausgebildete Blattform an die Oberfläche. Es stellt nunmehr sein Scheitelwachstum ein, bleibt also auf einer primitiven Stufe der Ausbildung stehen, seine äußeren Zellen bräunen sich und sein Rand verwächst schützend mit dem Stammteile und deckt die unter ihm weiter wachsende Stammknospe, bis es dann auch von dem nächsten Blatte zersprengt und in seiner schutzbringenden Aufgabe abgelöst wird. So zeigt die junge Rhizomknospe der Figur 47 das nicht median gezeichnete erste Blatt $\left(b_{3}\right)$ als äußere Decke, die bald von dem nächsten $\left(b_{2}\right)$ gesprengt werden kann und so fort. Schon die ersten Blätter der Keimpflanze treten in derselben Spiralstellung auf, wie die der älteren Pflanze, und haben auch gleiche Anlage und Entwicklung wie jene. Sie werden hier aber anfangs zu früh schon ihres deckenden Schutzes beraubt, an die Oberfläche geführt und dadurch in ihrem Scheitelwachstum, also in ihrer Ausbildung unterbrochen.

Wir untersuchen die Scheitelknospe einer Keimpflanze, z. B. einer solchen in der Entwicklung der Figur 48 näher. Die von außen in ihrer braunen Schattierung gesehenen Ringe sind die Ansatzstellen der ersten verbrauchten Blattschuppen, und soeben wird die letztere (etwa die dritte) von der darunter liegenden Schuppe gesprengt. Auch das letztere Blattorgan, das man am besten durch eine Präparation mit der Nadel freilegt, hatte nur eine sehr unvollkommene, etwa zweijährige Entwicklungsstufe erreicht und die Form eines stumpfkegelförmigen Deckels gewonnen, es deckte aber noch . ein zweites etwa einjähriges Blatt, und ein drittes befand sich in der Anlage.

In anderen Fällen kann eine gleichalterige Keimpflanze es schon auf ein kegelförmig hervortretendes äußeres Blatt, also auf eine höhere Entwicklungsform als die der flachen Schuppe gebracht haben.

Wir wissen von dem Studium der älteren Pflanze her, daß sie jährlich nur ein Blatt anlegt, welches zur vollständigen Entwicklung fünf Jahre nötig hat, von welcher Zeit es vier Jahre unterirdisch in der Scheide des nächstälteren Blattes wie in einem Futterale sich ausbildet. 
Von unserer Keimpflanze aber vermutet Hofmeister ${ }^{1}$ ), daß sie bereits in ihrer ersten Vegetationsperiode die ersten drei Wedel erzeuge, welche sich dann schon im zweiten Jahre ihres Daseins zu den ersten grünen und sporentragenden Wedeln entfalteten. Dagegen ist aber hervorzuheben, daß doch das geringe Bildungsvermögen des Rhizomscheitels der Keimpflanze ganz augenscheinlich wird. An .,sproßbildender Substanz" dürfte die jüngere Pflanze größeren Mangel haben als die ältere. Es kann daher unsere Keimpflanze auch nur jährlich ein Blatt erzeugen und noch dazu in bescheidenerer Form, als es die ältere Pflanze vermag, welches auch innerhalb einer Vegetationsperiode keine vollkommenere Entwicklungsstufe erreicht als bei der älteren Pflanze. Zur vollständigen Ausbildung eines sporentragenden Blattes auch in der bescheidenen Form der Figur 1 sind die gleichen fünf Jahre nötig. Die ersten "niederblattartigen Wedel" sind noch in unvollkommener Ausbildung, wenn sie ihr primitives Gehäuse, welches das nächstältere Blatt abgibt, sprengen. Sie übernehmen dann die äußere Deckung der Rhizomknospe und verlieren, wie schon hervorgehoben, außer der interkalaren Ausdehnung die weitere Entwicklungsfähigkeit. Man findet daher die ersteren Blätter, welche nur ein bis zwei Jahre eingeschlossen sich ausbilden konnten, als flache braune Schuppen den Scheitel deckend. Treffen wir sie äußerlich als lichtbraune oder weißliche spitzkonische Blattform an (Fig. $49 b z$ und Fig. 14 u. 15), so konnten sie sich zwei bis drei Jahre ungestört entwickeln. Nach solcher Zeit bringen sie es wohl zu einem grünenden Spitzchen und weiter bis auf die ersten Anlagen der gefiederten Spreite (Fig. 50 $a-c$ ). Nach der interkalaren Streckung finden sich die weißlichen Partien der Blätter mit braunen Zellen getüpfelt, welche wohl die Mutterzellen von unausgebildeten Spaltöffnunger darstellen mögen. Ein einfaches Bündel führt in jedes Erstlingsblatt. Unvollständige Rhizomknospen der Keimpflanze enthalten zwei, auch drei Blattformen. Erst aber, wenn die Blattscheide des äußeren Blattes vier Jahrgänge von Blattformen einschließt, ist sie vollständig und imstande, sporentragende sich an der Erdoberfläche entfaltende grüne Doppelblätter zu entwickeln.

Es ist auffallend, wie lange unsere Keimpflanze auf durch Chlorophyll gewonnene Assimilate verzichtet. Geboren wurde sie durch einen Gamophyten, dem die Assimilationsfähigkeit fehlte, und darauf kann sie ein, acht- bis zehnjähriges unterirdisches Dasein verbringen, ehe eins

1) Hofmeister, pag. 661 .

Flora 1906. 
ihrer Blätter über die Erdoberfläche tritt. Und wenn dies auch geschieht, ist die Assimilierung des einzigen Blattes mit seiner geringen Oberfläche, der mattgrünen Farbe und der kurzen, nur für das Frühjahr ausreichenden Lebensdauer sehr gering. Botrychium Lunaria ist fast ein Ganz-Saprophyt.

So lehrte uns denn diese Untersuchung ein eigenartiges Stammorgan kennen. Es wurde als ein unscheinbarer seitlicher Anhang an das ansehnliche Fußwurzelorgan des Keimlings angesetzt und gibt alsdann kräftigen Wurzeln, aber unscheinbaren Blattgebilden den Ursprung. Von den Ansatzstellen seiner Wurzeln anfangs umklammert, erhebt es sich erst allmählich über diese empor und kann dann zuweilen Internodien von fast ein Zentimeter Länge zwischen den Ansatzstellen einzelner Blätter bilden. Einen gesetzmäßigen Zusammenhang zwischen Wurzel- und Blattentwicklung, den Holle angibt, fand ich nicht bestätigt. Zuweilen wurden zwei Wurzeln auf einmal angelegt, was ich bei den Blättern nicht fand.

Die Eigenschaften der Gefäßbündel an Wurzeln, Stamm und Blatt sind genügend bekannt; und es ist daher eine nähere Betrachtung derselben hier überflüssig. Bemerken will ich nur, daß der hohle Gefäßzylinder des Stammes der Keimpflanze nach unten hin geschlossen erscheint und konisch der Ansatzstelle der beiden ersten Wurzeln der Keimpflanze aufgestellt ist (Fig. 47 u. 57).

c) Von der Verzweigung.

Das Rhizom von B. Lunaria ist meistens unverzweigt, und es kommat eine Verzweigung desselben äußerst selten vor. Röper $\left.{ }^{1}\right)$ beobachtete eine solche zuerst und stellte einige Fälle durch Zeichnungen dar, ohne sie aber näher zu untersuchen. $\mathrm{Holle}^{2}$ ) ist nur ein einziges verzweigtes Exemplar vorgekommen. Er nimmt eine endogene Entstehung derselben an und glaubt aus seiner Untersuchung schließen zu müssen, daß der Seitensproß in einer Blattachsel des Hauptsprosses inseriert sei. Auch der Seitensproß hatte von Anfang an reichlich Blätter und Wurzeln gebildet.

Ich habe etwa ein Dutzend einfach und mehrfach verzweigter Pflanzen unserer Art beobachtet und auch in Schnitten die Anlage einer Verzweigung angetroffen. Es scheinen namentlich jüngere Pflanzen eher geneigt zu sein, solche einzugehen als ältere.

1) Röper, Bot. Zeit. 1859.

2) Holle, Bot. Zeit. 1875, pag. 313. 
Derartige Verzweigungen gehen aus einer einzigen Oberflächenzelle des Rhizoms hervor. Bei jugendlichen Pflanzen streckt sich oft der Stamm und wächst mit seinen jüngsten Blattanlagen über die vorhergehenden empor, so daß in solchen Fällen ein entblößter Stammteil und somit freie Stammepidermis gewonnen wird. Fig. 57 zeigt links nur eine kurze Stufe des sich frei erhebenden Stammscheitels, aber es kommen auch solche von über einen Zentimeter Höhe vor.

An solcher freien Stammoberfläche traf ich eine peripherische Zellgruppe, im Längsschnitt gèsehen, an, wie sie die Zeichnung der Fig. 61 darstellt, die aus einer einzigen Oberflächenzelle entstanden und bei welcher die seitlichen Zellen (b) die mittleren $(s)$ eben umscheiden. Die letzteren mit auffallend dichtem Plasma und großen Zellkernen haben den Scheitel der Adventivknospe auszubilden, von welchen die obere Zelle schließlich die Form einer dreiseitigen Scheitelzelle annehmen wird. Fig. 62 zeigt eine gleiche Anlage in wenig weiterer Entwicklung. Die Umscheidung $(b)$ hat größere Fortschritte gemacht und die Zahl der den jungen Scheitel der Adventivknospe bildenden Zellen $(s)$ hat zugenommen. Auch die Zellen der Umgebung dieser Anlage gehen Teilungen ein, die namentlich auf einen frühzeitigen Anschluß der jungen Knospe an den Zentralzylinder des Rhizoms führen. Solchen Anschluß bildeten die in der Zeichnung angeführten Gefässzellen, wèlche nach oben auf die Scheide weisen. Es erinnert diese Art der Knospung an die embryonale Anlage des Rhizomscheitels und seine erste Umscheidung bei der Keimpflanze. Fig. 60 zeigt eine größere Keimpflanze mit 12 Wurzeln, die im Frühjahre aus dem Boden isoliert wurde, welche die erste Wurzel (wz) und den $\mathrm{Fuß}$ an ihr $(f)$ deutlich zu erkennen gibt,' bei welcher aber aus der gemeinsamen Scheide neben dem Blatte der Hauptknospe noch das einer Nebenknospe hervortritt. Diese Seitenknospe zeigte sich in der Entwicklung der Blätter sehr vorgeschritten und begann auch durch Anlage von Wurzeln sich selbständig zu machen. Diese hier so seltene Erscheinung der Verzweigung gleicht der Entwicklung der Seitenknospen bei Farnen, wie sie durch Mettenius und Prantl an einer Reihe Farne nachgewiesen worden ist. 


\section{Figuren - Erklärung.}

Tafel I, Fig. 1-42.

Fig. 1. Einige Prothallien und Keimpflanzen nebst ihrer Fundstelle in natürlicher Größe. $p$ die weißlichen Prothallien, $k$ Keimpflanzen, $k_{1}$ u. $k_{2}$ ältere Formen der letzteren.

Fig. 2-16. Prothallien und Keimpflanzen in dreifacher Größe.

Fig. 2 u. 3. Proth. in verschiedenem Alter.

Fig. 4-6. Proth. im Zusammenhange mit Keimpflanzen.

Fig. 7 u. 8. Junge freie Keimpflanzen mit ein er Wurzel.

Fig. 9-13. Keimpflanzen mit zwei, drei und vier Wurzeln und mit mehr oder weniger deutlicher Scheitelstelle $s, w_{1}$ Keim- oder erste Wurzel, die am hinteren Ende, dem Fuße, stets stumpfkeulig angeschwollen erscheint.

Fig. 16. Junge achtwurzelige Keimpflanze mit einem für die folgende Vegetationsperiode über die Erdoberfläche zu führenden ersten Sporenblatte.

Fig. 17-26. Prothallien und Keimpflanzen 16-fach vergrößert. Die Prothallien sind von der Oberseite gesehen gezeichnet. An ihren Seiten sollen die Kreise mit Quadranten die Archegonien; die auf der Rücken-Mitte punktierten Kreise, die Antheridien darstellen.

Fig. 17. Junges Prothallium. Fig. 18. Herzförmiges Prothallium.

Fig. 19 u. 20. Eiförmige Prothallien. em sich entwickelnde Embryonen.

Fig. 21-25. Prothallien im Zusammenhange mit Keimpflanzen.

Fig. 24 u. 25. Prothallien mit je zwei Keimpflanzen.

Fig. 26. Junge freie Keimpflanze. Sie stellt ein keuliges Wurzelgebilde dar. Am verborgenen Stammscheitel ist $w_{2}$ die Anlage der zweiten Wurzel. $f \mathrm{FuB}, w_{1}$ erste Wurzel.

Fig. 27. Ein vollständiges von oben gesehenes Prothallium, 60-fach vergrößert. $a$ der Anfang des Prothalliums (der Sporenpol desselben). $s$ die wachsende Region (der Scheitelpol). Die Antheridien an befinden sich auf der Rückenlinie. Zu beiden Seiten derselben die Archegonien ar. em ein Höcker mit einem Archegonium-Rudiment auf dem Gipfel, in dessen Innerem ein Embryo entwickelt wird. $h$ dunkelbraune Hyphen eines Pilzes, dessen Zweige in das Innere des Prothalliums eintreten.

Fig. 28. Medianer Längsschnitt durch ein junges Prothallium. a der Anfang (Sporenpol), s die wachsende Region (Scheitelpol), $b$ Bauch-, $r$ Rückenseite. an Antheridien, von denen das älteste entleert ist. $r h$ Rhizoide. $p$ Paraphysen. $h$ Hyphen eines eintretenden Pilzes. Das ganze Prothallium mit Ausnahme der Scheitel- und Rückenregion wird von einem endophytischen Pilze (en) bewohnt. Vrgr. 70.

Fig. 29. Medianer Längsschnitt durch die wachsende Region eines älteren Prothalliums. $m$ Scheitelmeristem. an erste Anlage eines Antheridiums, an und an ältere Entwicklungszustände desselben, $p$ Paraphyse, en endophytischer Pilz. Vrgr. 150.

Fig. 30. Querschnitt durch ein Prothallium mit hervorgetriebenen Antheridien (an) auf der Rückenmitte. ar altes verblühtes Archegonium, em junger Embryo, en endophytischer Pilz. Vrgr. 90. 
Fig. 31. Scheitelregion des Prothalliums von oben gesehen. $m$ Scheitelmeristem, an ein sich bildendes junges Antheridium, ar junge Archegonien, $r h$ Rhizoide, die an der Scheitelregion entwickelt werden. Vrgr. 150.

Fig. 32. Einige von dem Endophyten bewohnte Zellen des Prothalliums. $h$ Hyphen des Pilzes, $v$ Pilzklumpen, $k$ Fettkörper. Vrgr. 340.

Fig. 33. Zwei entleerte Antheridien von oben gesehen, das erste mit einer und das zweite. mit drei Öffnungen. Die punktierten Kreise geben den Umfang der bei tieferer Einstellung gesehenen Antheridien-Höhlung an. $p$ Paraphysen. Vrgr. 150.

Fig. 34. Eine Spermatozoiden-Mutterzelle und zwei Spermatozoiden. Vrgr. 550 .

Fig. 35. Reifes Archegonium kurz vor seiner Öffnung. Vrgr. 225.

Fig. 36. Befruchtetes Archegonium mit Eizelle kurz vor der Teilung. Vrgr. 225.

Fig. 37. Eizelle mit erster Teilung im Archegonium. Vrgr. 225.

Fig. 38. Vierzelliger Embryo. Vrgr. 225.

Fig. 39-41. Embryonen in der Richtung der Archegonium-Achse geschnitten. Vrgr. 225.

Fig. 42. Ein das Prothallium durchbrechender Embryo. Vrgr. 150.

Tafel II. Fig. $43-63$.

Fig. 43. Junge Keimpflanze im Zusammenhange mit dem Prothallium, dessen Zellen dicht mit Nährstoffen, namentlich Stärke, angefüllt sind. $f$ Fuß, zes Wurzelscheitel, $s$ Rhizomscheitel, an entleertes Antheridium, $p$ Paraphysen. Vrgr. 70.

Fig. 44. Die Sproßpartie der in Fig. 43 dargestellten Keimpflanze stärker vergrößert. $s$ Scheitelzelle mit den ersten Teilungen, von den angrenzenden Randzellen überwachsen, $c$ als rudimentäres Kotyledon zu deuten. Vrgr. 225.

Fig. 45. Ältere freie Keimpflanze mit stark ausgebildetem Fuße $f$. $w_{1}$ Wurzelscheitel der ersten Wurzel, $s$ Scheitel des Rhizoms mit der Anlage der zweiten Wurzel $w_{2}$. In den Zellen der Endophyt en. Vrgr. 52.

Fig. 46. Junge Keimpflanze im Längsschnitt. $w_{1}$ erste, $w_{2}$ zweite Wurzel, $f$ Fuß, $r$ Rhizom. Vrgr. 30.

Fig. 47. Das junge Rhizom der Keimpflanze von Fig. 46 in stärkerer Vergrößerung. $a$ Scheitelzelle des Vegetationspunktes desselben, $b_{1}$ erste, $b_{2}$ zweite Blattanlage, $b_{3}$ nicht medianes drittes Blatt. Vrgr. 150.

Fig. 48. Junge Keimpflanze mit fünf Wurzeln $\left(w_{1}-w_{5}\right)$. $s$ die wenig entwickelte Blattknospe. Vrgr. 16.

Fig. 49. Schematischer Durchschnitt einer Keimpflanze mit etwa sechs bis acht Wurzeln, von denen drei in derselben Ebene gezeichnet sind. $f$ Fuß, w erste Wurzel, $w$ Wurzeln, $s$ Rhizomscheitel, $b_{1}-b_{3}$ Blattentwicklungsformen. Vrgr. 6.

Fig. 50. Entwicklungsformen unvollkommen ausgebildeter Blätter von Keimpflanzen. Schwache Vrgr.

Fig. 51-54. Fig. 51 vierjährige, Fig. $52 a$ u. $b$ dreijährige, Fig. 53 zweijährige Blattformen älterer Pflanzen, Fig. 54 Querschnitt durch die Blattknospe. welcher die schraubenlinige Blattstellung anzeigt. Vrgr. 6.

Fig. 55. Scheitel des Rhizoms von oben gesehen. $a$ die dreiseitige Seheitelzelle desselben, $b_{1}$ Scheitelzelle mit junger Blattanlage. Vrgr. 340.

Fig. 56. Junges Blatt von oben gesehen. $b_{1}$ Scheitelzelle desselben, $i$ Blattinitiale des nächstjährigen Blattes. Vrgr. 225. 
Fig. 57. Längsschnitt durch eine Blattknospe. a Scheitelzelle des Rhizoms, $b_{1}$ des sich erhebenden erstjährigen Blatthöckers, $b_{2}$ Scheitelzellen des zweijährigen Blattes, welehes eben in Auszweigung begriffen ist.

Anm.: Die in verschiedenen Ebenen auftretenden Blätter sind in derselben Fläche gezeichnet. Vrgr. 225.

Fig. 58. Längsschnitt durch die verzweig̀ten Blatteile eines dreijährigen Blattes in der Entwicklung von Fig. $52 b$, in welchem die Scheitelzellen (b) deutlich erkennbar sind. Vrgr. $22 \overline{0}$.

Fig. 59. Schematischer Längsschnitt durch eine ältere Pflanze. w Wurzeln, s Rhizomscheitel, $b_{1}-b_{5}$ die Blätter in dem Jahresalter der beigegebenen Zahlen. Vrgr. 6.

Fig. 60. Ältere Keimpflanze mit etwa 12 Wurzeln, eine verzweigte Form darstellend. $f$ Fuß, $w_{1}$ erste Wurzel. Die beiden durchbrechenden Blätter deuten zwei Scheitelknospen an. Vrgr. 10.

Fig. 61 u. 62. Die ersten Entwicklungsstadien einer jungen Auszweigung. $s$ Scheitel derselben, $b$ Umscheidung der jungen Knospe. Vrgr. 225 u. 150.

Fig. 63. Junge Wurzelanlage im Rindengewebe des Rhizoms. w Wurzelscheitelzelle. Vrgr. 225. 